\title{
Spectroscopy Existing behind the Electro-Optical Properties with an Even-Odd Effect of nCB Liquid Crystal Molecules: A Theoretical Approach
}

\author{
N. KumaR ${ }^{a}$, P. Singh ${ }^{a}$, S. Chaudhary ${ }^{a}$, K.B. ThapA ${ }^{a}$, \\ P. UPADHYAY ${ }^{b}$, A.K. DWIVEDI ${ }^{c}$ AND D. KUMAR ${ }^{a, *}$ \\ ${ }^{a}$ Department of Physics, School of Physical and Decision Sciences, Babasaheb Bhimrao Ambedkar University, \\ Vidya Vihar, Raebareli Road, Lucknow (U.P.) 226025, India \\ ${ }^{b}$ Department of Physics, Sri J.N.P.G. College (KKC), Charbagh, Lucknow-226001, India \\ ${ }^{c}$ Department of Physics, M.L.K.P.G. College, Balrampur (U.P.) 271201, India
}

(Received November 27, 2019; in final form February 4, 2020)

In this work, we have studied the effect of the external electric field on the $\mathrm{nCB}$ series with an extension of the alkyl chain length of liquid crystal molecules. The stretching of $\mathrm{C}-\mathrm{C}$ and $\mathrm{C}-\mathrm{H}$ atom contributes to the anisotropy of polarizability for the new compound of the nCB series. The molecular polarizability is responsible for an even-odd effect of the optical parameters. The electric field is another method (alternative of temperature) to find out the optical parameters of the liquid crystal series. Under the impact of an external electric field, the order parameter and birefringence are expression of an even-odd effect. The transition temperature and birefringence enhance for the odd member and reduce for even members of the alkyl chain. The birefringence decreases with an even-odd effect while the order parameter increases with minor deviation.

DOI: 10.12693/APhysPolA.137.1135

PACS/topics: nCB liquid crystal, electric field, order parameter, birefringence, spectroscopy

\section{Introduction}

The electro-optical response depends on the physical property and molecular structure of the liquid crystal (LC) molecules. The cyanobiphenyl (nCB) LCs are highly polar, so it has very high thermal and electrochemical stability. The external electric field sufficiently affects the optical properties of rod shape LCs through the polarization of both ends of molecules. Under the effect of an external electric field, the one end of the $\mathrm{nCB}$ molecule has favorable charges. In contrast, the other end is negatively charged and formed an electric dipole. The director of the LC molecules will reorient along the direction of the applied external electric field [1-3]. The molecular polarizability increases with an increment of carbon atom number of the alkyl chain of the tail of the nCB LC molecules [4]. The family of the $\mathrm{nCB}$ LC first times synthesized by Gray et al. [5, 6] for the electro-optical application of the display technology. The $\mathrm{nCB} \mathrm{LC}$ is colorless and stable to the moisture that is the best feature of these molecules. When the $\mathrm{nCB} \mathrm{LC}$ having a unique property for the alkyl chain length is changed, then the molecular properties of the mesophase change $[5,6]$. The nCB LC molecules indicate on an evenodd effect under the extension of the alkyl chain. The optical polarizability of the $\mathrm{nCB}$ LC molecule follows the even-odd effect and gives the interchange polarizability.

*corresponding author; e-mail: dkclcre@yahoo.com
The order parameter also exhibits the even-odd effects for the even-odd number of the carbon atom of the alkyl chain in the tail of the ${ }_{n}$ CB LC $[7,8]$.

The birefringence is an electro-optical phenomenon used to find out the Kerr effect under the impact of the electric field. The birefringence is a fundamental property of the LC, which gives valuable information of LCs to use in various optical device applications. The electric field to the LC molecules has a rapid method to calculate birefringence and polarizability for the electro-optical application in the display technology. The electro-optical effect is used to study the pretransitional behavior (related to the order parameter) of the $\mathrm{nCB}_{\mathrm{LC}} \mathrm{C}$. The $\mathrm{nCB}$ LC has a positive Kerr constant because of the intense, permanent dipole moment and highly conjugated $\pi$-electrons [9-11]. The polarization of LC molecules induced by an applied electric field to the molecule depends on the direction of the applied field. The electric field corresponds to an essential parameter to find out the electro-optic property of the LC molecules [12, 13]. Rod shape LC molecules are less tilted with a comparison to the bent-core type LC molecules. The electrically induced tilt affects observed in several types of bent-core and hockey-stick type LC molecules [14].

The polarization and bending plane of the molecules are parallel in the absence of an electric field, and these will be perpendicular to the plane in the presence of an electric field. The smectic phase of the LC molecule tilted continuously with the increased electric field [15-18]. The switching behavior of the even-odd numbered carbon atom in the tail of the LC is different [19]. The notation 


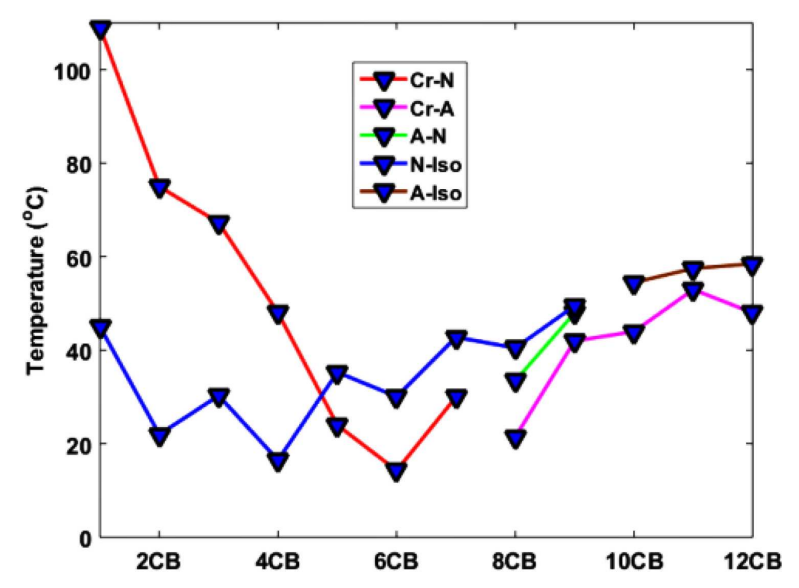

Fig. 1. Effect of temperature under the expansion of alkyl chain length. Red line indicates crystalline to nematic phase transition $(\mathrm{Cr}-\mathrm{N})$, blue line indicates nematic to isotropic phase transition ( $\mathrm{N}$-iso), green line indicates smectic A to nematic phase transition $(\mathrm{A}-\mathrm{N})$, pink line indicates crystalline to smectic A phase transition (Cr-A), brown line indicates smectic A to isotropic phase transition (A-Iso) [20].

of $\mathrm{nCB}$ refers to the number of carbon atoms in the alkyl chain of nCB LC molecules. The Kerr constant was measured with exploiting of birefringence under the impact of the electric field $E$. The birefringence $\Delta n$ is equal to the product of light wavelength $\lambda$, the Kerr constant $K$, and the square of the electric field. This can be expressed as $\Delta n=\lambda K E^{2}[21-24]$.

Karat and Madhusudana [25] reported that the even member of the $\mathrm{nCB}$ series makes a larger angle with the long molecular axis. It will reduce the anisotropy of the molecule and thereby minimizes the order parameter as well as nematic to the isotropic phase transition temperature. However, the odd member of the $\mathrm{nCB}$ series makes the least angle with the long molecular axis of the molecule. Thus, it will be enhancing the anisotropy of the molecule, thereby increasing the order parameter as well as transition temperature. The present work also reveals the order parameter and birefringence decreases for the even member. However, the order parameter and birefringence increase for the odd member of the $\mathrm{nCB}$ LC series. The $\mathrm{nCB}$ LC is a widely studied molecule, and all the optical parameters are readily available for comparison. Therefore, with the help of the described method, we can explain that the electric field is another method to achieve the all-optical properties and phase transitions, which is shown in Fig. 1 by the variation of temperature to the LC. Electric polarizability is the most crucial factor in finding optical parameters.

\section{Computational methodology}

All the molecules are optimized by the Gaussian 09 software package [26] with the help of density functional theory (DFT) method B3LYP [27, 28] and M062X [29] with $6-31 \mathrm{G}^{* *}$ basis set [30]. After the optimization of all the molecules, we have applied the electric field to the $\mathrm{nCB}$ LC along the molecular axis ( $x$-axis) and perpendicular (y-axis) to it from 0.0000 arb.u. to 0.2000 arb.u. at the interval of 0.0020 arb.u. After the applied electric field, we have calculated the molecular polarizability of the nCB LC molecules. The $x$-axis has been considered as extraordinary molecular polarizability $\left(\alpha_{e}\right)$, and $y$-axis has been considered as ordinary molecular polarizability $\left(\alpha_{o}\right)$. With the help of $\alpha_{e}$ and $\alpha_{o}$, we have calculated the order parameter, birefringence, and refractive index as per the given formula. The finite-field approach framework predicting the total molecular energy under the impact of the electric field is given below [31, 32];

$$
E=E_{0}-\mu_{i} F_{i}-\frac{1}{2} \alpha_{i j} F_{i} F_{j}-\frac{1}{6} \beta_{i j k} F_{i} F_{j} F_{k},
$$

where $E_{0}$ is the total energy in the absence of the electric field, while $F_{i}, \mu_{i}, \alpha_{i j}$, and $\beta_{i j k}$ are equivalent to the components of the electric field, dipole moment, polarizability, and first-order hyperpolarizability, respectively. Directions are specified along with the subscripts $(i, j, k) \in(x, y, z)$. The $\alpha, \beta, \mu$, and $\Delta \alpha$ can be expressed as numerical differentiation with an electric field of magnitude 0.002 arb.u. The respective equations are given below [33-35]:

$$
\begin{aligned}
& \alpha= \frac{1}{3}\left(\alpha_{x x}+\alpha_{y y}+\alpha_{z z}\right), \\
& \beta= {\left[\left(\beta_{x x x}+\beta_{x y y}+\beta_{x z z}\right)^{2}+\left(\beta_{y y y}+\beta_{x x y}+\beta_{y z z}\right)^{2}\right.} \\
&\left.+\left(\beta_{z z z}+\beta_{x x z}+\beta_{y y z}\right)^{2}\right]^{1 / 2}, \\
& \mu=\left(\mu_{x}^{2}+\mu_{y}^{2}+\mu_{z}^{2}\right)^{1 / 2}, \\
& \Delta \alpha=\sqrt{\frac{\left(\alpha_{x x}-\alpha_{y y}\right)^{2}+\left(\alpha_{y y}-\alpha_{z z}\right)^{2}+\left(\alpha_{z z}-\alpha_{x x}\right)^{2}}{2}} \\
& \Delta \tilde{\alpha}=\alpha_{\mathrm{e}}-\alpha_{\mathrm{o}}, \quad \Delta \tilde{\alpha}=S \Delta \alpha,
\end{aligned}
$$

where $\tilde{\alpha}$ is the mean isotropic polarizability, $S$ is the order parameter

$$
S=\frac{\alpha_{\mathrm{e}}-\alpha_{\mathrm{o}}}{\alpha_{e}+\alpha_{\mathrm{o}}} \text {. }
$$

Birefringence $\Delta n$ is expressed as

$$
\Delta n=\frac{\left(\alpha_{\mathrm{e}}-\alpha_{\mathrm{o}}\right)}{6.3631}\left[R^{3}-\left(\frac{2 \alpha_{\mathrm{o}}+\alpha_{\mathrm{e}}}{20.244}\right)\right]^{-1},
$$

where $R$ is the radius of the liquid crystal molecule. Magic angle $(\theta)$ is given

$$
\theta=\cos ^{-1}\left(\frac{2 S+1}{3}\right)
$$

and refractive index $n$ by

$$
\begin{aligned}
& \alpha=\frac{2 \alpha_{\mathrm{o}}+\alpha_{\mathrm{e}}}{3}, \\
& \gamma_{\mathrm{e}}=\alpha+\frac{2\left(\alpha_{\mathrm{e}}-\alpha_{\mathrm{o}}\right)}{3 S}, \quad \gamma_{\mathrm{o}}=\alpha-\frac{\left(\alpha_{\mathrm{e}}-\alpha_{\mathrm{o}}\right)}{3 S},
\end{aligned}
$$




$$
\begin{array}{r}
n_{\mathrm{e}}=\frac{7}{2 \sqrt{10}}+\frac{(2 \sqrt{10} / 5) \pi N \alpha}{1-\frac{4 \pi N \alpha}{3}} \\
+\frac{(4 \sqrt{10} / 15) \pi N S\left(\gamma_{\mathrm{e}}-\gamma_{\mathrm{o}}\right)}{1-\frac{4 \pi N \alpha}{3}} \\
n_{\mathrm{o}}=\frac{7}{2 \sqrt{10}}+\frac{(2 \sqrt{10} / 5) \pi N \alpha}{1-\frac{4 \pi N \alpha}{3}} \\
-\frac{(2 \sqrt{10} / 15) \pi N S\left(\gamma_{\mathrm{e}}-\gamma_{\mathrm{o}}\right)}{1-\frac{4 \pi N \alpha}{3}} \\
n=\frac{7}{2 \sqrt{10}}+\frac{(2 \sqrt{10} / 5) \pi N \alpha}{1-\frac{4 \pi N \alpha}{3}},
\end{array}
$$

where $N=1$ is the number of liquid crystal molecules studied with the variation of temperature and external electric field to find out the different phases of LC series. The temperature range of the $\mathrm{LC}$ is $300 \mathrm{~K}$ to $400 \mathrm{~K}$ used to study the phases of the LC series, as shown in Fig. 1.

\section{Results and discussion}

\subsection{Effect of the temperature} with an extension of alkyl chain length

This subsection describes the different phase transition of $\mathrm{nCB}$ liquid crystal with the variation of temperature. The red line is indicating the crystalline to the nematic phase transition from $1 \mathrm{CB}$ to $7 \mathrm{CB}$. The $8 \mathrm{CB}$ to $12 \mathrm{CB}$ showing crystalline to smectic phase $\mathrm{A}$ transition are indicated by the pink line. The $8 \mathrm{CB}$ to $9 \mathrm{CB}$ showing smectic A to nematic phase transition are indicated by the green line. Nematic to the isotropic phase transition of $1 \mathrm{CB}$ to $9 \mathrm{CB}$ shown by the blue line and lastly smectic $\mathrm{A}$ to the isotropic phase transition of $10 \mathrm{CB}$ to $12 \mathrm{CB}$ are indicated by brown line. The temperature variation expresses the even-odd effect for the phase transition of the $\mathrm{nCB}$ series $[20,36]$, which is also shown by the application of the electric field on the nCB series. Dalmolen et al. [37], Dunmur et al. [38] and Lin et al. [39] reported that the $\mathrm{nCB}$ series expresses an even-odd effect under the impact of the temperature of LC. The nCB LC molecules reveal an even-odd effect under the influence of the temperature by an extension of alkyl chain length. Karat and Madhusudana [25] already reported that even member of the $\mathrm{nCB}$ series is making a larger angle with the long molecular axis. Thus, it will reduce the anisotropy of the molecule and thereby minimizes the nematic to the isotropic phase transition temperature. However, the odd member of the $\mathrm{nCB}$ series is making a lower angle with the long molecular axis of the molecule. Thus, it will be enhancing the anisotropy of the molecule, thereby increasing the transition temperature shown in Fig. 1. The $\mathrm{C}-\mathrm{C}$ symmetric scissoring in the benzene ring is responsible for crystalline to the nematic phase transition. The IR frequency corresponding to the absorbance continually increases from $1 \mathrm{CB}$ to $7 \mathrm{CB}$. However, the absorbance decreases for the $8 \mathrm{CB}$ because

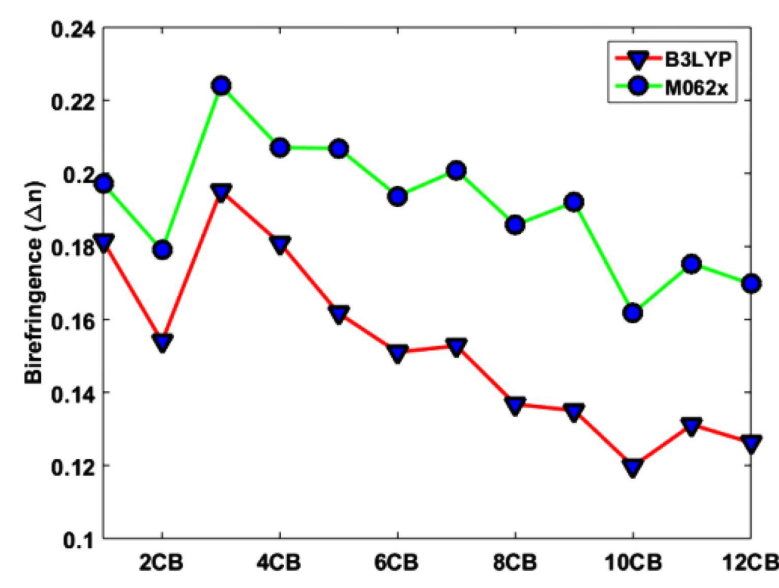

Fig. 2. Calculated birefringence of $\mathrm{nCB}$ series under the effect of the external electric field with an extension of the alkyl chain length using B3LYP (red line) and M062X (green line) methods.

it is expressed crystalline to the smectic A phase transition indicates by the pink line. The stretching of the $\mathrm{C}-\mathrm{C}$ and $\mathrm{C}-\mathrm{H}$ atoms has contributed to the anisotropy of polarizability for the new compound as a comparison with the previous molecule [40].

\subsection{Birefringence}

We have calculated birefringence with the help of (2) under the external electric field with an extension of the alkyl chain length. Coles [12] reported that the birefringence is decreasing under the influence of the external electric field with an extension of alkyl chain length. In present work, the birefringence is decreasing continuously with an extension of the alkyl chain, as shown in Fig. 2. Dunmur et al. [38] have reported that under the impact of temperature, the dielectric anisotropy and birefringence decreased. The $\mathrm{nCB}$ series shows an evenodd effect with the extension of the alkyl chain length. Under the influence of an electric field, the birefringence of nCB LC molecules decreases with the even-odd effect, as shown in Fig. 2. According to $\mathrm{Wu}$ [41], the birefringence of $5 \mathrm{CB}$ is 0.22 , while we obtained the value of 0.20 . Although the birefringence steadily decreases from $1 \mathrm{CB}$ to $5 \mathrm{CB}$, it does not show the even-odd effect. Still, after the 5CB LC, the birefringence exhibits the perfectly evenodd effect express by the DFT methods B3LYP, as shown in Fig. 2. However, the even-odd effect is expressed by the DFT method M062X. The odd carbon atom number of the alkyl chain has a higher birefringence as compared to the even member of the alkyl chain. The birefringence decreases with an increase of carbon atom numbers of the alkyl chain length. The $\mathrm{C}-\mathrm{H}$ asymmetric stretching corresponds to IR absorbance in the benzene ring, shows an improvement for the odd member, and falls for the even member of the alkyl chain. As the 10CB LC is an even member of the $\mathrm{nCB}$ series, its IR absorbance shows increasing behaviour. 


\subsection{Order parameter}

Karat and Madhusudana [25] reported that the even members of the $\mathrm{nCB}$ series make a larger angle with the long molecular axis. As a result, it will reduce the polarizability of the molecules and thereby minimize an order parameter. Our studies show also that 10CB LC reduces larger molecular polarizability, and it is abruptly decreasing. Horn [36] reported that the order parameter of $5 \mathrm{CB}$ and $8 \mathrm{CB}$ is 0.61 and 0.67 , respectively. In turn, calculations made by us for the order parameter of $5 \mathrm{CB}$ and $8 \mathrm{CB}$ indicate the values of the value of 0.60 and 0.71, respectively. Dalmolen et al. [37] and Sherrell and Crellin [42] reported that the order parameter increases with an extension of alkyl chain length.

In the present work, an order parameter continuously increases with an extension of the alkyl chain of the $\mathrm{nCB}$ LC molecules, as shown in Fig. 3. The order parameter also exhibits the even-odd effect with minor deviation. Park [43] reported that the order parameter is determined from $\mathrm{C}-\mathrm{N}$ and $\mathrm{C}-\mathrm{C}$ stretching. The order parameter continually increases because of the $\mathrm{C}-\mathrm{N}$ atom stretching, and $\mathrm{H}$ atom rocking of benzene ring corresponding to IR absorbance continuously increases. The $\mathrm{C}-\mathrm{H}$ atom asymmetric stretching in the alkyl chain is steadily increasing, which is also responsible for the increment in the order parameter. The order parameter with an extension of the alkyl chain goes continuously to the crystalline state because the range of liquid crystal is 0.3 to 0.8 . Sen et al. [44] reported that under the effect of temperature, the order parameter of $5 \mathrm{CB}$ is 0.62 . In the present work, the order parameter calculated under the effect of the electric field is 0.60 . The order parameter is an optical property of the $\mathrm{nCB}$ LC. Based on the order parameter, we can examine the molecular behavior of the $\mathrm{nCB} \mathrm{LC}$ molecules. The $\mathrm{C}-\mathrm{H}$ asymmetric stretching of the benzene ring at the frequency of $1532 \mathrm{~cm}^{-1}$ is showing higher IR absorbance for odd members and

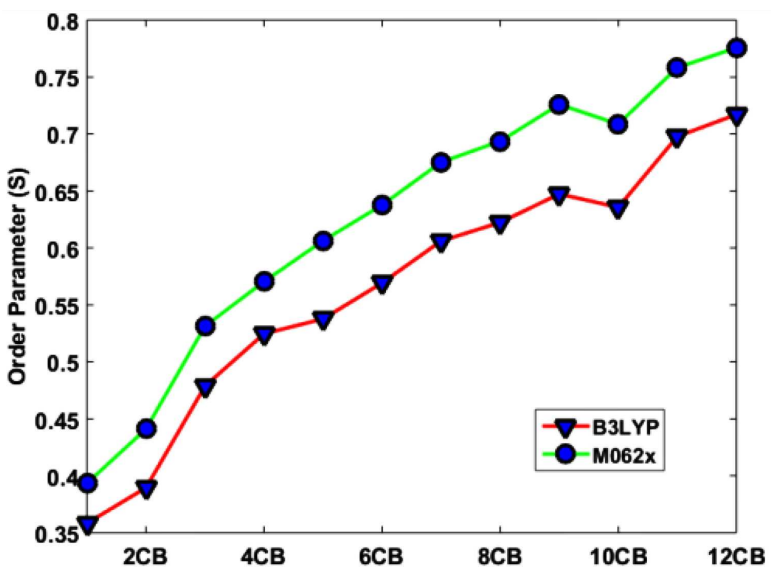

Fig. 3. Calculated order parameter $S$ of nCB series under the effect of the external electric field with an extension of the alkyl chain length using B3LYP (red line) and M062X (green line) methods.

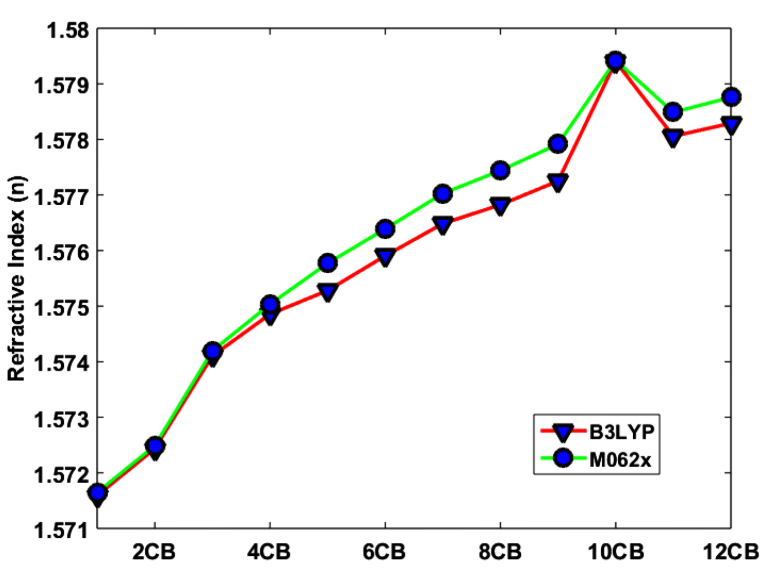

Fig. 4. Calculated refractive index of nCB series under the effect of the external electric field with an extension of the alkyl chain length using B3LYP (red line) and M062X (green line) methods.

lower IR absorbance for even members of the alkyl chain. The $\mathrm{C}-\mathrm{C}$ symmetric scissoring in the benzene ring is responsible for molecular phases. At the frequency of $2266 \mathrm{~cm}^{-1}$, the $\mathrm{C}-\mathrm{N}$ atom stretching corresponding to absorbance continually increases, which is accountable for an increment of the order parameter.

\subsection{Refractive index}

Chirtoc et al. [45] reported that under the impact of temperature, the refractive index is increasing with an extension of alkyl chain length. This behaviour appears continually, as shown in Fig. 4 . The $\mathrm{C}-\mathrm{H}$ atom asymmetric stretching in the alkyl chain is continuously increasing, which is also responsible for an increment of refractive index. In the 10CB LC, the refractive index abruptly increases. In case of the $\mathrm{C}-\mathrm{H}$ asymmetric stretching corresponding to an IR absorbance it is growing instead of decreasing in the benzene ring. Thus, the refractive index does not express any even-odd effect. The $\mathrm{H}$ atom rocking in both benzene ring corresponding to IR frequency $1532 \mathrm{~cm}^{-1}$ increases only in 10CB LC, which is responsible for abruptly increased refractive index. In fact, we have observed that the IR spectrum of 10CB LC also indicates the absorbance due to the $\mathrm{H}$ atom rocking in both benzene ring.

\section{Conclusion}

In this work, we have investigated the effect of the electric field on the $\mathrm{nCB}$ LC series, which shows an evenodd effect in optical applications. It has been found that the electric field is another method to find out the optical parameters of the nCB series. The optical parameters are obtained using derived equations from Vuk's theory [46]. The birefringence of $\mathrm{nCB}$ series decreases continuously and shows an even-odd effect with the extension of the alkyl chain length of the $\mathrm{nCB}$ LC molecules. The order parameter of the $\mathrm{nCB}$ is steadily increasing with 
an extension of the alkyl chain length of the nCB series. The $\mathrm{C}-\mathrm{C}$ and $\mathrm{C}-\mathrm{H}$ atoms stretching contributes to the anisotropy of polarizability. The stretching of $\mathrm{C}-\mathrm{C}$ and $\mathrm{C}-\mathrm{H}$ atoms corresponds to higher IR absorbance for odd members and lowers IR absorbance for even members of the $\mathrm{nCB}$ series. The birefringence and order parameter of the $\mathrm{nCB}$ LC molecules are inversely proportional to each other. Under the effect of the electric field, the order parameter, and birefringence are different for the different numbers of carbon atom numbers. The birefringence, order parameter, and transition temperature of $\mathrm{nCB}$ series exhibit a higher value for odd carbon members, which corresponds to the $\mathrm{C}-\mathrm{H}$ asymmetric stretching of the benzene ring. Our results help to investigate the optical parameters of liquid crystals, which can be obtained through electric field and temperature. It also explains the correlation between microscopic parameters to mesoscopic parameters of LC, which can be obtained by variation of external electric field and temperature of liquid crystals. The motivation of the work studies the reason behind the even-odd effects obtained by the variation of temperature and electric field. The temperature and electric field both parameters are showing the oddeven effect in the LC series. Hence, the interesting part of the study is the correlation between the electric field and temperature to cause the even-odd effect in the LC series. This strategy is useful for the electro-optical effect of future molecules.

\section{Acknowledgments}

Narinder Kumar is very thankful to the University Grants Commission (UGC) New Delhi for providing fellowship (RGNF). We are also very grateful to Dr. Anoop Ayyappan (IIT KGP, WB) for providing computational help. P. Singh and S. Chaudhary are thankful to UGC for providing a non-NET fellowship.

\section{References}

[1] J. Ananthaiah, R. Sahoo, M.V. Rasna, S. Dhara, Phys. Rev. E 89, 022510 (2014).

[2] P.G. De Gennes, The Physics of Liquid Crystal, Clarendon Press, Oxford 1974, p. 1.

[3] S. Chandrasekhar, Rep. Prog. Phys. 39, 613 (1976).

[4] R.J.W. Le Fevre, B.P. Rao, Aust. J. Chem. 10, 1465 (1958).

[5] G.W. Gray, K.I. Harrison, J.A. Nash, Electron. Lett. 9, 130 (1973).

[6] G.W. Gray, A. Mosley, J. Chem. Soc. Perkin Trans. 2, 97 (1976).

[7] M.I. Caparand, E. Cebe, J. Comput. Chem. 28, 2140 (2007).

[8] J.R. Lalanne, B. Lemaire, J. Rouch, C. Vaucamps, A. Proutiere, J. Chem. Phys. 73, 1927 (1980).

[9] J. R. Lalanne, J.C. Rayez, B. Duguay, A. Proutiere, R. Viani, J. Chem. Phys. 81, 344 (1984).
[10] M.J. Aroney, Angew. Chem. 16, 663 (1970).

[11] T.W. Stinson, J.D. Litster, Phys. Rev. Lett. 25, 503 (1970).

[12] H.J. Coles, Mol. Cryst. Liq. Cryst. 49, 67 (1978).

[13] D.A. Dunmur, Mol. Phys. 23, 109 (1972).

[14] P. Sathyanarayana, S. Radhika, B.K. Sadashiva, S. Dhara, Soft Matter 8, 2322 (2012).

[15] A. Eremin, M. Floegel, U. Kornek, S. Stern, R. Stannarius, Phys. Rev. E 86, 051701 (2012).

[16] E.G. Hanson, Y.R. Shen, G.K.L. Wong, Phys. Rev. A 14, 1281 (1976)

[17] P. Palffy-Muhoray, D.A. Dunmur, Mol. Cryst. Liq. Cryst. 97, 337 (1983).

[18] P. Singh, K.B. Thapa, N. Kumar, K. Pal, D. Kumar, Mater. Res. Express 6, 066209 (2019).

[19] M.J. Aroney, W.P. McPherson, R.K. Pierens, J. Mol. Struct. 69, 289 (1980).

[20] D. Dunmur, A. Fukuda, G.R. Luckhurst, Physical Properties of Liquid Crystals: Nematics, Institute of Electrical Engineers, London 2001.

[21] N. Asgharian, Z.A. Schelly, Biochim. Biophys. Acta 1418, 295 (1999).

[22] D.P. Shelton, Rev. Sci. Instrum. 64, 917 (1993).

[23] H. Ibrahim, W. Haase, Mol. Cryst. Liq. Cryst. 66, 189 (1981).

[24] N. Vieweg, C. Jansen, M.K. Shakfa, M. Scheller, N. Krumbholz, R. Wilk, M. Mikulics, M. Koch, Opt. Exp. 18, 6097(2010).

[25] P.P. Karat, N.V. Madhusudana, Mol. Cryst. Liq. Cryst. 36, 51 (1976).

[26] M.J. Frisch, G.W. Trucks, H.B. Schlegel et al., Gaussian 09 (now Gaussian 16), Gaussian Inc., Wallingford (CT) 2016.

[27] A.D. Becke, J. Chem. Phys. 98, 5648 (1993).

[28] C. Lee, W. Yang, R.G. Parr, Phys. Rev. B 37, 785 (1988).

[29] Y. Zhao, D.G. Truhlar, Theor. Chem. Acc. 120, 215 (2008).

[30] P.J. Hay, W.R. Wadt, J. Chem. Phys. 82, 299 (1985).

[31] H.D. Cohen, C.C.J. Roothaan, J. Chem. Phys. 43, S34 (1965).

[32] A.D. Duckingharn, Adv. Chem. Phys. 12, 107 (1967).

[33] A. Kumar, A.K. Srivastava, S.N. Tiwari, N. Misra, D. Sharma, Mol. Cryst. Liq. Cryst. 681, 23 (2019).

[34] H.J. Deuling, Mol. Cryst. Liq. Cryst. 19, 123 (1972).

[35] H.S. Kitzerow, Mol. Cryst. Liq. Cryst. 202, 51 (1991).

[36] R.G. Horn, J. Phys. (France) 39, 105 (1978).

[37] L.G.P. Dalmolen, S.J. Picken, A.F. de Jong, W.H. de Jeu, J. Phys. (France) 46, 1443 (1985).

[38] D.A. Dunmur, M.R. Manterfield, W.H. Miller, J.K. Dunleavy, Mol. Cryst. Liq. Cryst. 45, 127 (1978).

[39] Z. Shu-Lin, P. Zheng-Yu, W. Jin, S. Tie-Han, W. Nai-Qiang, Mol. Cryst. Liq. Cryst. 91, 295 (1983). 
[40] E. Galbiati, G. Zerbi, J. Chem. Phys. 84, 3509 (1986).

[41] S.T.-Wu, Appl. Opt. 26, 3434 (1987).

[42] P. Sherrell, D. Crellin, J. Phys. Colloq. 40, C3-211 (1979).

[43] W.-S. Park, J. Korean Phys. Soc. 37, 331 (2000).

[44] Miss S. Sen, P. Brahma, S.K. Roy, D.K. Mukherjee, S.B. Roy, Mol. Cryst. Liq. Cryst. 100, 327 (1983).

[45] I. Chirtoc, M. Chirtoc, C. Glorieux, J. Thoen, Liquid Cryst. 31, 229 (2004).

[46] J. Li, S.-T. Wu, J. Appl. Phys. 96, 6253 (2004). 